\title{
Molecular characterization of three forms of putative membrane-bound progestin receptors and their tissue-distribution in channel catfish, Ictalurus punctatus
}

\author{
Yukinori Kazeto, Rie Goto-Kazeto, Peter Thomas ${ }^{1}$ and John $\mathbf{M}$ Trant \\ Center of Marine Biotechnology, University of Maryland Biotechnology Institute, 701 East Pratt Street, Baltimore, Maryland 21202, USA \\ ${ }^{1}$ Marine Science Institute, University of Texas at Austin, 750 Channelview Drive, Port Aransas, Texas 78373, USA
}

(Requests for offprints should be addressed to John M Trant; Email: trant@umbi.umd.edu)

\begin{abstract}
Membrane-bound progestin receptors (mPR) were recently cloned and characterized as a new class of steroid receptors that transduce cell-signals through alteration of MAP kinase- and cAMP-dependent pathways. To further develop our understanding of this new class of steroid receptors, we characterized the cDNAs and genes of the $\alpha, \beta$ and $\gamma$ forms of the channel catfish mPRs (IpmPR). The predicted $\alpha$ and $\beta$ proteins have $49 \%$ sequence identity, whereas they only have $30 \%$ and $27 \%$ identities, respectively, with the $\gamma$ form. Furthermore, IpmPR $\alpha$ and IpmPR $\beta$ genes have similar structures featuring intronless coding regions, while IpmPR $\gamma$ gene is composed of 8 exons and 7 introns. The 5'-flanking region of each IpmPR gene differs, but each contains putative transcriptional regulatory elements of factors known to influence reproductive physiology and endocrine disruption, for example, responsive elements for cAMP and steroids and the recognition sites for steroidogenic factor- 1 and for the aryl hydrocarbon receptor. The IpmPR $\alpha$ gene was detected in all the tissues tested with relatively greater expression in brain, pituitary, muscle and testis. The expression of IpmPR $\beta$ was much lower than that of IpmPR $\alpha$ and the transcript was predominantly observed in brain, pituitary, ovary and testis. In contrast, the IpmPR $\gamma$ transcript was mainly detected in gill, ventral aorta, intestine, and trunk kidney. In conclusion, all the structural features of the IpmPRs strongly suggest that the closely related $\alpha$ and $\beta$ forms are distantly related to the $\gamma$ form. Additionally, regulatory features of the $5^{\prime}$-flanking regions and the differences in tissue-specific expression of each IpmPR gene suggest that they are involved in different endocrine functions in catfish.
\end{abstract}

Journal of Molecular Endocrinology (2005) 34, 781-791

\section{Introduction}

Steroid hormones play important roles in many physiological processes, including reproduction. The classic mechanism of steroid action involves alteration of gene transcription, which typically is a relative slowly process, by diffusing into the cell and binding to ligand-activated transcription factors belonging to the nuclear steroid receptor superfamily (Beato 1989, Tsai \& O’Malley 1994). Steroids have also been shown to exert rapid effects in many target tissues that are initiated at the cell surface by binding to steroid membrane receptors and frequently occur via nongenomic mechanisms (Falkenstein et al. 2000, Losel \& Wehling 2003, Simoncini \& Genazzani 2003). However, the identities of the steroid membrane receptors mediating most of these nonclassical actions remain unknown, and there is evidence for the existence of both unique receptors and nuclear receptor-like forms on the plasma membranes of steroid target cells (Bagowski et al. 2001, Losel \& Wehling 2003, Xu et al. 2003).

Meiotic maturation of fish and amphibian oocytes is one of the most well-characterized nongenomic steroid actions mediated by membrane-bound steroid receptors at both the biochemical and cytological levels (Yamashita et al. 2000). The precise mechanism for the activation and/or stabilization of the maturationpromoting factor by the maturation-inducing steroid (MIS) appears to differ among species. For example, the MIS-receptor (MIS-R), which in fish is a membranebound progestin receptor ( $\mathrm{mPR}$ ), appears to be coupled to an inhibitory G-protein $(\mathrm{G} \imath)$ in three fish species: rainbow trout, Atlantic croaker and spotted seatrout, (Yoshikuni \& Nagahama 1994, Thomas et al. 2002, Zhu et al. 2003a), whereas in zebrafish and an amphibian, Xenopus, it is likely that progestins activate a stimulatory G-protein (Gallo et al. 1995, Kalinowski et al. 2004). However, many other processes initiated through activation of the membrane-bound MIS-R, such as a decrease in intracellular cAMP levels, have been shown to be conserved (Yamashita et al. 2000).

Until recently, despite intensive research in several laboratories, efforts to characterize MIS-Rs in vertebrates at the molecular level have been unsuccessful (Maller 1998). For the first time in any species, a novel 
$\mathrm{mPR}$ cDNA was recently isolated and characterized in a teleost fish, the spotted seatrout, and several lines of evidence suggest it is the MIS-R in this species (Zhu et al. 2003a). Subsequently, three forms of $\mathrm{mPR}(\alpha, \beta$ and $\gamma)$ have been identified in several other species of vertebrates, from fish to mammals (Zhu et al. 2003b). The finding that antisense oligonucleotides for both $\mathrm{mPR} \alpha$ and $\beta$ blocked MIS induction of oocyte maturation in zebrafish provided an initial indication that both mPRs may serve as the MIS-Rs in this species (Zhu et al. 2003a, Thomas et al. 2004). However, there are still many unanswered questions concerning the precise physiological roles of the three members of this new class of steroid receptors in fish and other vertebrates.

In this paper, we report the characterization of the channel catfish, Ictalurus punctatus, cDNAs encoding three forms of $\mathrm{mPR}(\mathrm{mPR} \alpha, \beta$ and $\gamma)$, their genomic organization, the structures of their $5^{\prime}$-flanking regions, and their tissue distributions. Our findings clearly suggest that the catfish mPRs are related to the seatrout $\mathrm{mPR}$ and that the highly related $\alpha$ and $\beta$ forms are involved in catfish reproductive physiology, but their roles as MIS-Rs remain to be demonstrated.

\section{Materials and methods}

\section{Animals}

All fish were maintained and tissue samples were taken in accordance with protocols approved by the University's Institutional Animal Care and Use Committee

\section{Oligonucleotides and DNA sequencing}

Oligonucleotide PGR primer synthesis and nucleotide sequence analysis were provided by the BioAnalytical Services Laboratory at the Center of Marine Biotechnology. Nucleotide sequences were determined with the PRISM Cycle sequencing kit using an ABI Model 377 DNA Sequencer (PE Applied Biosciences, Foster City, CA, USA).

\section{Cloning of cDNA encoding putative channel catfish mPRs (IpmPRs)}

Total RNA was extracted from ovary, testis and posterior kidney of channel catfish using Trizol Reagent (Life Technologies Inc, Gaithersburg, MD, USA), from which poly $(\mathrm{A})+-\mathrm{RNA}$ was purified with Straight A's mRNA isolation system (Novagen, Madison, WI, USA). The poly(A)+-RNA was reverse-transcribed into cDNA using PowerScript (BD Bioscience, Palo Alto, CA, USA) after priming with a clamped oligo $(\mathrm{dT})$ primer. Three sets of degenerate primers were designed to separately amplify each type of mPR by PCR (Table 1). The channel catfish mPR (IpmPR) $\alpha$ and $\beta$ cDNA fragments were amplified from ovary and testis cDNA with mPRS1 and A1, and mPRS2 and A2, respectively, while the isolation of an IpmPR $\gamma$ cDNA fragment was carried out using kidney cDNA with mPRS3 and A3 primers. The resultant PCR products were inserted into a TA cloning vector (pDrive; Qiagen, Valencia, CA, USA) and sequenced. Subsequently, 5'- and $3^{\prime}$-RACE of testis cDNA amplified the sequences of the $5^{\prime}$ and $3^{\prime}$ ends of each cDNA encoding IpmPR $\alpha$ and $\beta$ using the SMART RACE cDNA Amplification kit (BD Bioscience) according to the manufacturer's instructions. Posterior kidney cDNA was used for the RACE PCR cloning of the cDNA for the $\gamma$ form. Gene-specific primers, mPRA4 and S4, were used for 5'- and 3'-RACE to obtain full length cDNA of the $\alpha$ form. Due to low transcript abundance, nested RACE PGRs were required for the isolation of the $\beta$ form cDNA. The first 5'- and 3'-RACE PCRs were conducted with mPRA5 and S5 and were followed by a second set of RACE PCRs using mPRA6 and S6, respectively. RACE PCRs for the $\gamma$ form were carried out using mPRA7 and S7. Finally, to isolate amplicons, including the entire open reading frames for each type of IpmPR, PGRs were conducted with the following three sets of primers: mPRS 8 and A8, mPRS9 and A9, and mPRS 10 and A10 for IpmPR $\alpha, \beta$ and $\gamma$, respectively, using a proof reading PfuTurbo DNA polymerase (Stratagene, La Jolla, CA, USA). The resultant amplicons were cloned and sequenced as described above.

Phylogenetic analysis of the mPRs characterized to date was carried out by the neighbor-joining method using the Clustal W and njplot programs (Saitou \& Nei 1987). Potential transmembrane domains, $\mathrm{N}$-glycosylation sites and phosphorylation sites in mPRs were predicted by analyses posted on the web sites, www.ch.embnet.org/software/TMPRED_form.html (Hofmann \& Stoffel 1993), www.cbs.Dtu.dk/services/ NetNGlyc/ and www.cbs.Dtu.dk/services/NetPhos/ (Blom et al. 1999), respectively.

\section{Characterization of genomic organization and the 5 '-flanking regions of the $\mathrm{mPRs}$}

Genomic DNA was extracted from catfish brain using a Blood \& Cell Culture DNA Starter Kit (Qiagen). A catfish GenomeWalker library was constructed with a Universal GenomeWalker Kit (BD Bioscience) according to the manufacturer's protocol. 'Genome walking' PCRs using the GenomeWalker library was carried out using Advantage-GC genomic polymerase and the gene-specific primers (mPRA11-A16) listed in Table 1 in order to characterize the $5^{\prime}$-flanking region of the three types of IpmPR genes. Typical PCRs using genomic 
Table 1 Oligonucleotides and fluorescent probes used for PCR

Nucleotide sequence

Name

mPRS1 5'-GTVTACCARTWYGGNAGYGC-3'

mPRA1 5'-TGGAAGANYTGRTGNCCYTG-3'

mPRS2 5'-CTGTTCRNGARCTNTAYAT-3'

mPRA2 5'-GGCACNGGRCANGARAARAA-3'

mPRS3 5'-ACYCTSAAYATDTGGACNCA-3'

mPRA3 5'-CACACRTGRAANAGYGRTG-3'

mPRS4 5'-ACAGTTCACAGCAGGGTGCACCGACCC-3'

mPRA4 5'-GCCCAGGGAACCAGCACTCTGGATGAG-3'

mPRS5 5'-CGGCTCTGACCTACCTCACCTGTAGCAC-3'

mPRA5 5'-GATCTTCCTGCGCAGCGGGTATGGACG-3'

mPRS6 5'-GTGCCCACTAGCCTGGCGTACATGTTGG-3'

mPRA6 5'-CAGAAGCGTAGTAGCACAGCAGGTACCCC-3

mPRS7 5'-GGCACCTGGACGTTTCGACTTAGTAGGCC-3'

mPRA7 5'-CAGGTGCCAGCCTCTCGGGCAGATGTG-3'

mPRS8 5'-CACCTTCATCATGGCGACCGTGGTGATGG-3'

mPRA8 5'-CGCCTTCCCAAGTCTCCTCACTCCTGC-3'

mPRS9 5'-CGCTGGACATAACCCAGAAAGGAGCTGATC-3'

mPRA9 5'-CCCTCCAGAACTGGAGGTAAACTCCC-3'

mPRS10 5'-GCAGTCTCACCAGCAACCTGGAACCTG-3'

mPRA10 5'-AAGCTCTAGTTGGTGCCCTTAGTTC-3'

mPRA11 5'-GACCTGAAGCATATACATGGTGAAAGCG-3'

mPRA12 5'-TGTTCCAGCGGGTGAGGCTCCTGTCA-3'

mPRA13 5'-AGGTCAACTGTCCATGTAGTGCACTG-3'

mPRA14 5'-GTAACTGTCGTGGTTCACAAGATGAAGCC-3'

mPRA15 5'-GTACATTGAAGACGGGAGGCAGCTTGA-3'

mPRA16 5'-CAGGTTCCAGGTTGCTGGTGAGACTGCC-3'

mPRS11 5'-AGAGCGTATAAAAGGTCGTGCATAA-3'

mPRA17 5'-CACGGTCGCCATGATGAA-3'

mPRP1 FAM-5'-CTGATTGGCTCTTGAAAAGATCCCAGAAAGTTC-3'-TAMRA

mPRS12 5'-GAGGCCGAATCCCTCCAT-3'

mPRA18 5'-CAGACGGCCTAAAACTCCACTT-3'

mPRP2 FAM-5'-CTGGACATAACCCAGAAAGGAGCTGATCACT-3'-TAMRA

mPRS13 5'-GGCAGTATTCAACACTGCGTTATC-3'

mPRA19 5'-GTGAAGGCTAAAACCCGTAGCA-3'

mPRP3 FAM-5'-TGGCCTGTTACTCCAGATTTGCCGAA-3'-TAMRA

mPRS14 5'-TCTTCACTGCCTGGCTGAGATA-3'

MPRA20 5'-GAACAGCCGCCCAATCTG-3

mPRP4 FAM-5'-TCCTCACCATGGCGACGGTTGTG-3'-TAMRA

mPRS15 5'-GAAGAGCTACTTTCTGCATAGAGTTTCA-3'

mPRA21 5'-AGACGAGGCAACCAGTTCGA-3'

mPRP5 FAM-5'-TGCAGCAGCTGGGTCAGTTACCACAC-3'-TAMRA
Use and its target DNA

IpmPR $\alpha$ cDNA fragment

IpmPR $\alpha$ cDNA fragment

IpmPR $\beta$ cDNA fragment

IpmPR $\beta$ cDNA fragment

IpmPR $\gamma$ cDNA fragment

IpmPR $\gamma$ cDNA fragment

3'-RACE for IpmPR $\alpha$ cDNA

5'-RACE for IpmPR $\alpha$ cDNA

3'-RACE for IpmPR $\beta$ cDNA

5'-RACE for IpmPR $\beta$ cDNA

3'-RACE for IpmPR $\beta$ cDNA

5'-RACE for IpmPR $\beta$ cDNA

3'-RACE for IpmPR $\gamma$ cDNA

5'-RACE for IpmPR $\gamma$ cDNA

ORF of IpmPR $\alpha$ cDNA

ORF of IpmPR $\alpha$ cDNA

ORF of IpmPR $\beta$ cDNA

ORF of IpmPR $\beta$ cDNA

ORF of IpmPR $\gamma$ cDNA

ORF of IpmPR $\gamma$ cDNA

1st Genome walking for IpmPR $\alpha 5^{\prime}$-flanking region 2nd Genome walking for IpmPR $\alpha 5^{\prime}$-flanking region 1st Genome walking for IpmPR $\beta$ 5'-flanking region 2nd Genome walking for IpmPR $\beta 5^{\prime}$-flanking region 1st Genome walking for IpmPR $\gamma 5^{\prime}$-flanking region 2nd Genome walking for IpmPR $\gamma$ 5'-flanking region TaqMan of IpmPR $\alpha$

TaqMan of IpmPR $\alpha$

Probe of IpmPR $\alpha$ for TaqMan

TaqMan of IpmPR $\beta$ form

TaqMan of IpmPR $\beta$ form

Probe of IpmPR $\beta$ for TaqMan

TaqMan of IpmPR $\gamma$

TaqMan of IpmPR $\gamma$

Probe of IpmPR $\gamma$ for TaqMan

TaqMan of DpmPR $\alpha$ form

TaqMan of DpmPR $\alpha$ form

Probe of DpmPR $\alpha$ for TaqMan

TaqMan of DpmPR $\beta$

TaqMan of DpmPR $\beta$

Probe of DpmPR $\beta$ for TaqMan
DNA or 'genome walking' PCRs were also conducted using proof-reading DNA polymerases (PfuTurbo DNA polymerase (Stratagene), Advantage-GG genomic polymerase or Advantage 2-GC polymerase (BD Biosciences)) and appropriate primers based on the cDNA sequences for each mPR under the thermal cycling conditions recommended by the manufacturer. Intron/exon boundary sites were determined by comparing the genomic sequence with the respective cDNA sequence.

Putative transcriptional factor binding sites located on the 5'-flanking region of each IpmPR gene were identified with the aid of analyses posted on the web site, http://motif.genome.ad.jp/ with an $80 \%$ cut off score.
Additionally, consensus core sequences for transcriptional factor binding sites potentially related to reproductive physiology or endocrine disruption, i.e., cAMP responsive element (CRE: TKACGTMA), estrogen responsive element (ERE: AGGTCANNNTG ACCT), glucocorticoid/progestin/androgen responsive element (GRE/PRE/ARE: AGAAGANNNTGTTCT), steroidogenic factor-1 binding site (SF-1: TCAAGG TYA) and the responsive element for aryl hydrocarbon receptor and its nuclear transfer (AhR/Arnt: TYGC GTG), were manually searched using software, DNASIS-Mac v3.5. All potential elements are more than $80 \%$ identical to their corresponding core sequences. 


\section{Gene-specific expression of catfish and zebrafish mPRs}

Total RNA $(1 \mu \mathrm{g})$ prepared from various tissues (i.e., parts of the brain (telencephalon, cerebellum, diencephalon, hypothalamus and hindbrain), pituitary, gill, ventral aorta, intestine, muscle, heart, spleen, liver, posterior kidney, head kidney and ovary) from a female adult catfish and from testis from a male adult catfish was primed with a clamped oligo(dT) primer and reverse-transcribed using M-MLV reverse transcriptase (Life Technologies Inc). Real-time, quantitative RTPCR, (rtqRT-PCR; PE Applied BioSystems) was employed to measure transcript abundance of each IpmPR gene. The primers and probes used for each gene are listed in Table 1. The primer/probe sets for IpmPR $\alpha$ and IpmPR $\beta$ were mPRS11, A17 and mPRP1, and mPRS12, A18 and mPRP2, respectively. The set of primer/probes for the $\gamma$ form was mPRS13, A19 and mPRP3. Transcript abundance of each mPR was normalized to the abundance of catfish $\beta$-actin (GenBank accession number AY555575; Kazeto et al. 2003). The same type of analysis was conducted in zebrafish to examine the tissue-specific distribution of the zebrafish mPR (DrmPR) $\alpha$ and $\beta$ genes. The primer/probe sets to measure transcript abundance of DrmPR $\alpha$ and DrmPR $\beta$ by TaqMan were mPRS14, A20 and mPRP4, and mPRS15, A21 and mPRP5, respectively (Table 1). The detailed procedure to design and synthesize the primer/ probe sets, and to validate similar rtqRT-PCR assays has been described elsewhere (Trant et al. 2001).

\section{Identification of putative mPRs and their genomic organization in human and Fugu}

BLAST searches of human and Fugu genome databases were conducted with each form of catfish mPRs using the analyses posted on the web site of The Wellcome Trust Sanger Institute (www.sanger.ac.uk) in order to identify cognate cDNAs and their genomic organization, and to compare them with the corresponding IpmPRs characterized in this report.

\section{Results}

\section{Isolation of the cDNAs encoding IpmPRs}

PCR-based isolation of cDNAs encoding putative IpmPR $\alpha$ (GenBank accession number AY533540), $\beta$ (GenBank accession number AY533541) and $\gamma$ (GenBank accession number AY533542) were completed with the aid of 5'-and 3'-RACE PCRs. The length of the nucleotide sequences of IpmPR $\alpha, \beta$ and $\gamma$ are 2303, 3487 and $1186 \mathrm{bp}$, respectively. Coding regions of $\alpha, \beta$ and $\gamma$ forms consist of 1062, 1068 and 1017 bp encoding 354, 356 and 339 amino acid residues, respectively. The degree of amino acid sequence identity between the $\alpha$ form and the $\beta$ and $\gamma$ forms is $49 \%$ and $30 \%$, respectively, while the $\beta$ and $\gamma$ forms have $27 \%$ sequence identity

\section{Identification of potential cognate cDNAs of mPRs from human and Fugu genome databases}

A BLAST search of the human and Fugu genome databases was carried out using the deduced amino acid sequences of catfish mPRs to identify the homologous genes. Six putative mPRs, including $\alpha$ (Ensembl ID. SINFRUP00000149401) and $\gamma$ (ID. SINFRUP 00000127469) forms reported previously (Zhu et al. 2003b) were retrieved from the Fugu database. Fugu mPR $\beta$ (ID. SINFRUP00000160848) was also newly identified. Three other cognate forms named Fugu mPRlike protein (FmPRLP 1-3) appear to be novel forms. FmPRLP 1 (SINFRUP00000145530) and FmPRLP 2 (SINFRUP00000145528) are structurally closely related and share $78 \%$ identity. The predicted amino acid sequences of FmPRLP 1 and 2 show approximately $50 \%, 40 \%$ and $30 \%$ identities to Fugu $\mathrm{mPR} \alpha, \beta$ and $\gamma$, respectively. The degree of identity between FmPRLP 3 (SINFRUP00000156015) and FmPRLP 1 and 2 is 44\% and $38 \%$. FmPRLP 3 shares $39 \%, 46 \%$ and $25 \%$ homology with Fugu mPR $\alpha, \beta$ and $\gamma$, respectively. Three forms of $\mathrm{mPR} \alpha$ (ENSP00000329071), $\beta$ (ENSP00000302785) and $\gamma$ (ENSP00000260380) were identified by this search in the human genome as reported previously (Zhu et al. 2003b).

\section{Structural and phylogenetic analysis of IpmPRs}

Phylogenetic analysis of the mPR-related proteins clearly shows that the catfish mPRs are clustered together with their respective mPR types amongst the fish representatives and that the $\gamma$ forms appear to be highly diversified from the other two groups (Fig. 1). FmPRLP 1 and 2 appear to be phylogenetically derived from $\mathrm{mPR} \alpha$, although they are not tightly clustered with fish mPRas. In contrast, FmPRLP 3 is clearly placed together with fish mPR $\beta$, especially the Fugu form.

The amino acid sequences of each IpmPR and their related forms from the other species are aligned in Figures 2-4. The deduced amino acid sequence of IpmPR $\alpha$ showed high homology with other fish forms (higher than 70\%) and 49-54\% sequence identity with mammalian forms and FmPRLP 1 and 2. IpmPR $\beta$ has $72 \%$ sequence identity to zebrafish $\mathrm{mPR} \beta$ and $48-55 \%$ identity to other mPR $\beta$-related forms, including FmPRLP 3. Fugu mPR $\gamma$ shares the closest identity to IpmPR $\gamma \quad(71 \%)$. Transmembrane and lipophilicity analyses identified seven potential transmembrane domains in all $m P R \alpha$ - and $\beta$-related forms except for FmPRLP 1 and zebrafish mPR $\beta$ that appeared to lack the sixth and second transmembrane domains, 


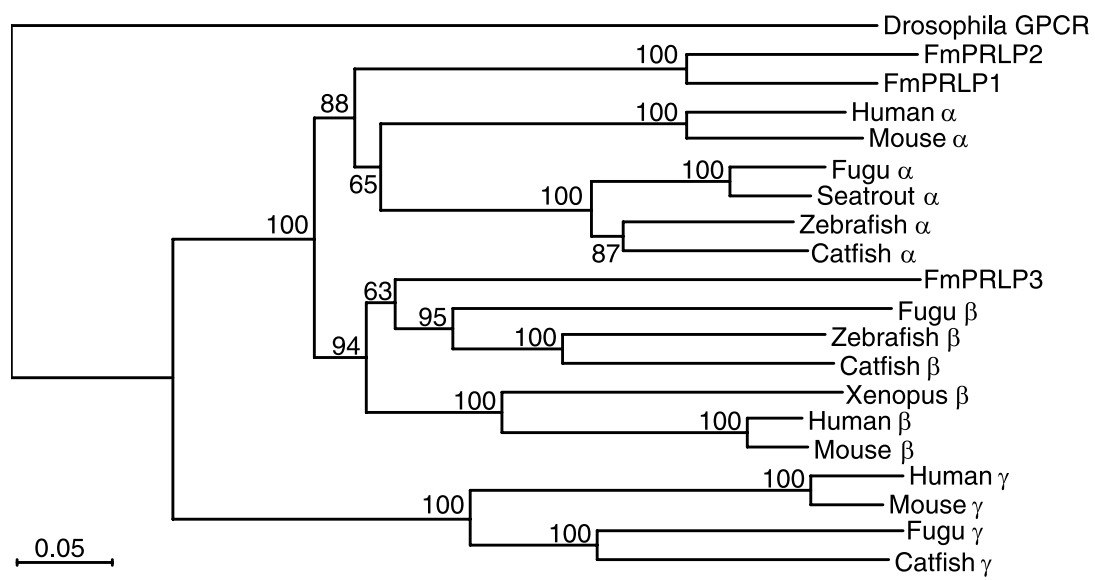

Figure 1 Phylogenetic analysis of mPR-related proteins. The analysis was performed by the neighbor-joining method using full-length protein sequences. The numbers beside the branches indicate bootstrap values from 100 replicates.

respectively. IpmPR $\gamma$ possesses seven potential transmembrane domains like $\mathrm{mPR} \alpha$ and $\beta$, but other $\gamma$ forms only have five or six potential transmembrane domains by this analysis.

One potential $\mathrm{N}$-linked glycosylation site $(\mathrm{N} \times \mathrm{S} / \mathrm{T})$ located in the N-terminal extra-cellular region was identified in the $\alpha$ and $\gamma$ forms, but not in the $\beta$ forms. Several phosphorylation sites that are conserved among the fish forms and among all forms of each type of mPR were identified, however all sites are located in extracellular regions or transmembrane domains. Two highly conserved regions were identified in all forms of $\mathrm{mPR} \alpha$, $\beta$ and $\gamma$ (Figs 2-4). One region, composed of sixteen amino acid residues that span the $\mathrm{N}$-terminal region and first transmembrane domain and includes a potential $\mathrm{N}$-linked glycosylation site, is highly conserved and the identity between this region of IpmPR $\alpha$ and those of all other $\mathrm{mPR}$ forms is over $70 \%$. The second conserved region (fifteen amino acids) is located on the putative boundary of the third cytoplasmic loop and sixth transmembrane domain in the $\alpha$ and $\beta$ forms, while this region corresponds to the fourth extracellular region of IpmPR $\gamma$. This conserved region in IpmPR $\gamma$ is over $60 \%$ identical to the other forms of $\mathrm{mPR}$.

\section{Comparison of IpmPRa, $\beta$ and $\gamma$ gene structure to human and Fugu mPRs}

The genomic organizations of each IpmPR, as determined by PCR-based strategy, are illustrated in Figure 5. Intron/exon junctions of each IpmPR gene were identified by comparison with the corresponding IpmPR cDNA sequence. Interestingly, both the IpmPR $\alpha$ and $\beta$ genes do not possess any introns on their coding regions. The IpmPR $\alpha$ gene is over $4331 \mathrm{bp}$ with a large single intron (only 2061 bp has been sequenced) interrupting the $5^{\prime}$-UTR. The untranslated exon I is $577 \mathrm{bp}$ in length, whereas exon II contains 33 nucleotides of the $5^{\prime}$-UTR as well as the entire coding region (1062 bp) and 3'-UTR (598 bp). The genomic organization of the IpmPR $\beta$ gene is comparable to that of the IpmPR $\alpha$ gene, except for an additional intron in the 5'-UTR. The entire sequence of this gene has been determined to be $5118 \mathrm{bp}$ in length. First and second exons of the IpmPR $\beta$ gene encode $582 \mathrm{bp}$ and $125 \mathrm{bp}$ of the 5'-UTR, respectively. Exon III consists of the remaining $9 \mathrm{bp}$ of the $5^{\prime}-\mathrm{UTR}$, the whole coding region (1068 bp) and the $3^{\prime}$-UTR (1681 bp). Intron I and II are 756 bp and $897 \mathrm{bp}$, respectively. In contrast, the structure of IpmPR $\gamma$ gene spanning approximately $15.7 \mathrm{kbp}$ is quite different from the other IpmPR forms and includes 8 exons (with a non-coding exon I) and 7 introns. The sizes of introns are 263, 1727, 7585, 243, 1883, 1397 and $1431 \mathrm{bp}$, respectively. All the nucleotide sequences of the intron/exon boundary sites are consistent with the classical GT/AG rule (Breathnach \& Chambon 1981) involved in the recognition for intron splice site.

The structures of the $\mathrm{mPR} \alpha$ and $\beta$ genes of human and Fugu share the basic characteristics of their respective IpmPR genes, i.e., these genes do not contain introns in their open reading frames. Furthermore, human and Fugu mPR $\gamma$ genes possess 6 introns in the coding region like the IpmPR $\gamma$ gene and the position for all intron/exon junctions are identical to those in the IpmPR $\gamma$ gene. Additionally, there are no introns in the coding regions of FmPRLP 1 and 2 genes that are structurally cognate to $\mathrm{mPR} \alpha$ at the phylogenetic level. In contrast, the FmPRLP 3 gene is structurally different from all three IpmPRs (i.e., 4 intron/exon junctions that are not identical to those in $\operatorname{mPR} \gamma$ genes) even though the predicted protein is highly similar to $\operatorname{mPR} \beta$. 


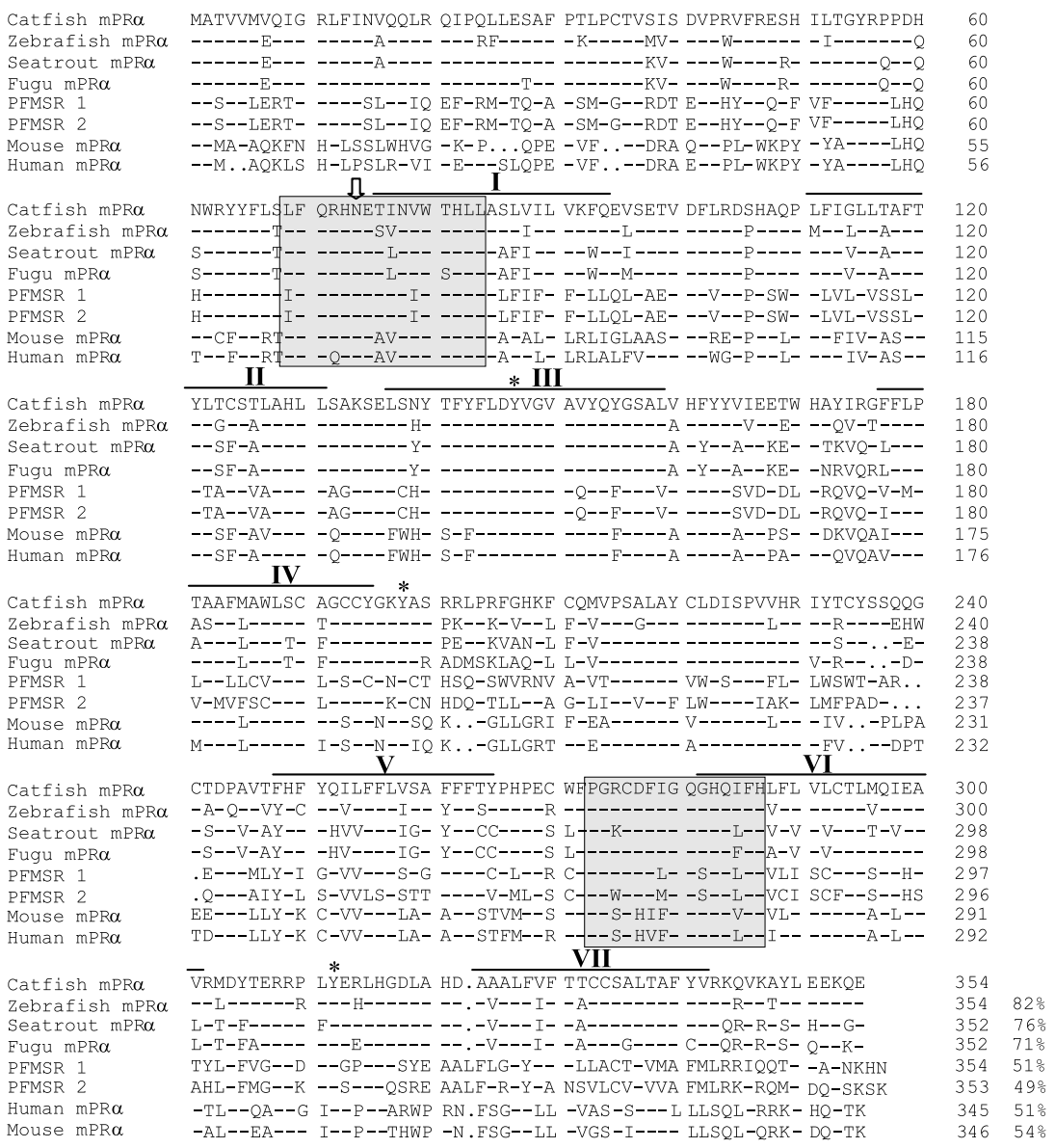

Figure 2 Alignment of the deduced amino acid sequence of IpmPR $\alpha$ with the $\mathrm{mPR} \alpha$ related proteins of other animal species. Dashes indicate residues that are identical to IpmPR $\alpha$. Dots indicate gaps introduced to facilitate alignment. Hatched boxes indicate highly conserved regions in all mPR-related proteins. The arrow shows the potential $\mathrm{N}$-linked glycosylation site. I-VII indicate the putative transmembrane domains. ${ }^{*}$, the putative phosphorylation sites.

\section{Structure of $5^{\prime}$-flanking regions of the IpmPR genes}

Sequence analysis of the GeneWalker amplicons generated $3045 \mathrm{bp}$ (GenBank Accession number AY587768), $3701 \mathrm{bp}$ (GenBank accession number AY587769) and 3382 bp (GenBank accession number AY587770) of unique sequences representing the 5 '-flanking regions of IpmPR $\alpha, \beta$ and $\gamma$ genes, respectively. Figure 6 is a schematic diagram showing potential transcription regulatory elements located within the $3 \cdot 0 \mathrm{kbp}$ upstream of the end of the 5 '-UTR for each IpmPR gene. The full lengths of the $5^{\prime}$-UTRs were determined by sequence analysis of $5^{\prime}$-RACE amplicons. A TATA box was identified within $90 \mathrm{bp}$ of the end of the $5^{\prime}$-UTR of each IpmPR. The $5^{\prime}$-flanking region of the IpmPR $\alpha$ gene contained the consensus sequences of two CREs, two binding sites for SF-1, five former-half sequences of GRE/PRE/AREs and two latter-half sequences of EREs. The regulatory 5 '-flanking region of the IpmPR $\beta$ gene showed the consensus sequences of three binding sites for SF-1, four half-GRE/PRE/AREs, three half-EREs, a binding site for AhR/Arnt and a binding site for early growth response-4 (Egr-4). Four half palindromic sequences for GRE/PRE/AREs, a binding site for SF-1, a binding site for AhR/Arnt, and an AhR/Arnt binding site overlapped with a recognition site for Egr-2 and Egr-3 were located on the $5^{\prime}$-flanking region of IpmPR $\gamma$ gene.

\section{Tissue distribution of transcripts of mPRs in catfish and zebrafish}

Differential tissue-distribution of the transcripts for mPRs in catfish (Fig. 7A) and for zebrafish (Fig. 7B) was determined by sensitive real-time, quantitative RT-PCR 
Catfish mPR $\beta$ Zebrafish mPR Fugu mPRP PFMSR 3 Xenopus mPR Mouse mPR $\beta$ Human $\mathrm{MPR} \beta$

Catfish mPR $\beta$ Zebrafish mPR

Fugu mPR $\beta$

PFMSR 3

Mouse mPR $\beta$

Human mPR $\beta$

Catfish mPR $\beta$ Fugu mPR PFMSR 3 Xenopus mPR $\beta$ Human mPR $\beta$

Catfish mPR $\beta$ Zebrafish mPR Fugu mPR $\beta$ PFMSR 3 Xenopus mPR $\beta$ Mouse MPR $\beta$ Human mPR $\beta$

Catfish mPR $\beta$ Zebrafish mPR Fugu $\mathrm{mPR} \beta$ PFMSR 3 Xenopus mPR $\beta$ Mouse mPR $\beta$ Human mPR $\beta$

Catfish mPR $\beta$ Zebrafish mPR $\beta$ Fugu mPR Xenopus mPR $\beta$ Monopus mPR $\beta$ Mouse mPR $\beta$ Zebrafish mPR

MSSGVLGRLS TLTLNIKQVV GLPHLSPSLH .SLPSLQATV EVSNVPSLFR EPYIQSGYRP

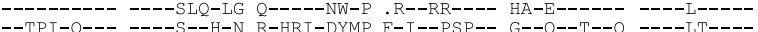
PSV-D RL---CPP-- GDTE--P--- K-F-V----TTAI-ECI- --SISFQ-LR R--RE. . .-E GGTTKMPL-- TD-D--R--- ----QT----TTAI-E--- --SMSGQ-LR R--KI.... E EG--KMPC-- PETD--O--- -.--HA---TTAI-E--- --SVSGQ-LR R--KI... -E DG--KMPC-- PETD--Q--- ----RT---VGQPWHCYAL S SLFQRHNELI NVWSHLIGVP AVLLRFWLFA ..ATRSLTLD AS.SLPLFLY $\begin{array}{ll} & 116 \\ & \end{array}$ $\begin{array}{lll} & 117\end{array}$

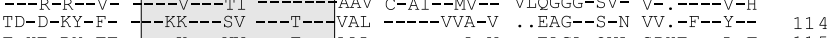
T-HE-RY-FF ----K---VV ---T---AAL -------A-V .. EAGA-OWA SPHT---L-F 115 T-HE-RY-FF ----K---VV ---T---AAL -------A-A ...EAEA-PWA STHS---L-F 115 $\frac{1}{\text { TLSALTYLTC STLAHLLQSH }} \stackrel{*}{*} \frac{*}{\text { SELSHYSLFF LDYVGVAVYQ YGSALAHYFY CAEEEWKQSI }} 176$ V-

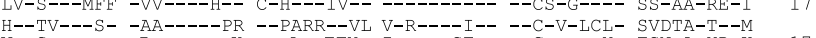
V--SI---- -L------K ------TFY- V-----S--- ------F-- SSDQA-YE.L 174 I--SI----- - IV------K -----TFY- V-----S--- ------F-- SSDQA-YD.R 174 VGALFLPGAA LLGWLSCASC CFAKLHYRRP YPLRRKIFQI VPTSLAYMLD ISPVALRLAR 236 --V------- M-A------- -YS-FR---- --FH---C-- I------L-- -----H--LT 236 --L----- -----LG- -IS-FR-Q-- --WQ---C-L I---A--L-- ----VH--FT 237

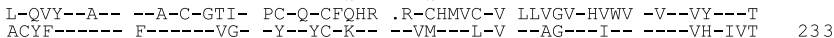

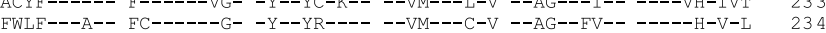
FWLF---A-- FC------G- -Y--YR---- --VM---C-V --AG--FI-- -- VI-H-V-L 234 RPC ... HEPV LVLHALQVAF FLMAAFFFSC PVPERFFPGK CDIVGHGHQI FHIFLVMCTM 293

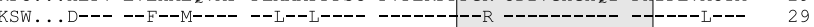
VSWS. .Q--S -PF----I-C --LS------ -I------Q --F--Q---- --VL-SL--I 295

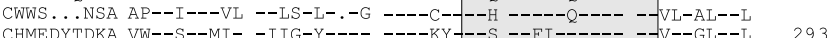

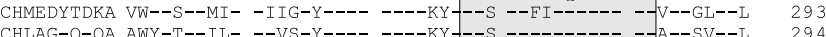

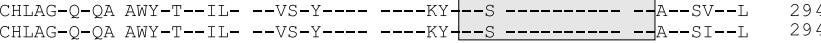
CQMEAVFKDF LVQRSLVVHV HGEWSLWLAV GSFFVLVLCS ILTAARMRQR VQRQLQKERLKSE 35 CQMEAVFKDF LVQRSLVVHV HGEWSLWLAV GSFFVLVLCS ILTAARMRQR VQRQLQKERLKSE 356 - L- -M-R-- - - HQQS-DA ---HFIL--G ----L----- ----VL--GA $-----R-K D \quad 35272 \%$ F-L--L-Q-Y ARR-DTM-E- F--GR--W-C VGLPL-F-GC LI-LLTMKH -KNHRTTTKKDK $35755 \%$ S-I--I-I-Y OTROEVFSAR YSSNYTIMCC A---I-I--- TF--VYA-R- IKEK-ARKE- 35348 S-L$\begin{array}{llll}\text { S-L--ILL-Y QGRHEIFLQR --PL-VYS-C L-----AA-- AA--SLL-HK -KDR-I-KDS } & 354 & 54 \% \\ \text { S-L--I-L-Y QGRQEIFLQR --PL-VHM-C L---F-AA-- AA---LL-HK -KAR-T-KSD } & 354 & 55 \%\end{array}$

Figure 3 Alignment of the deduced amino acid sequence of IpmPR $\beta$ with the $\mathrm{mPR} \beta$ related proteins of other animal species. The key for the symbols is shown in Figure 2 legend.

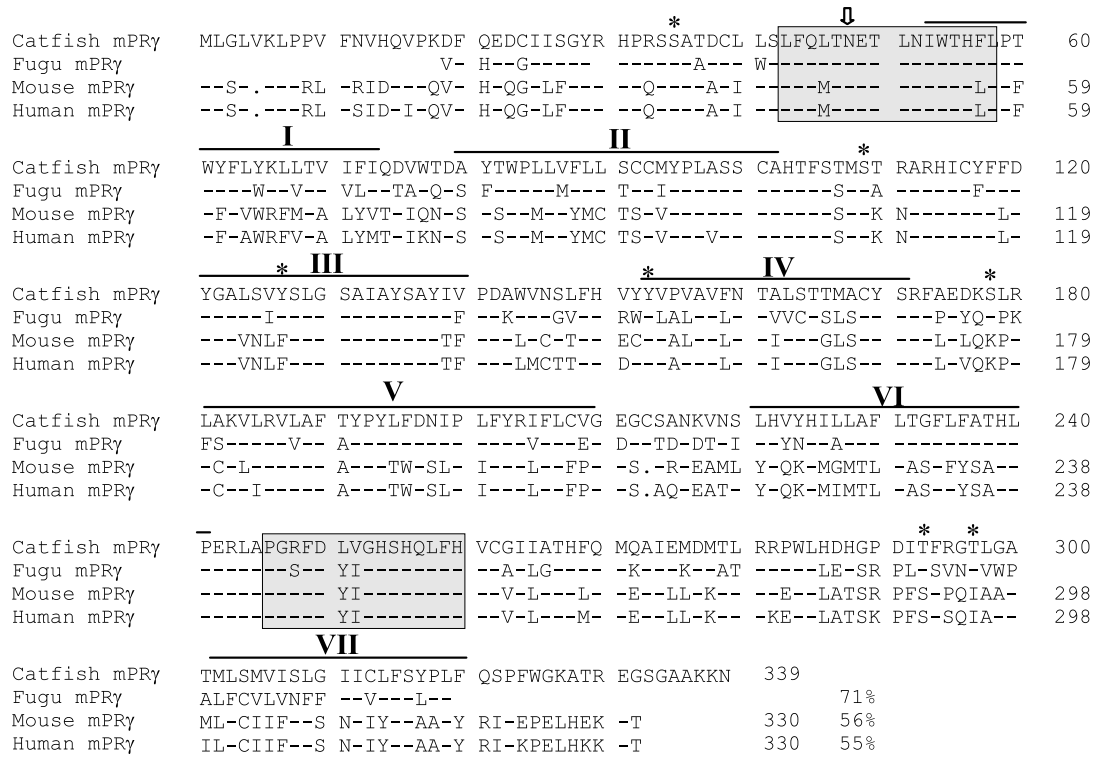

Figure 4 Alignment of the deduced amino acid sequence of IpmPR $\gamma$ with the mPR $\gamma$ related proteins of other animal species. The key for the symbols is shown in Figure 2 legend. 


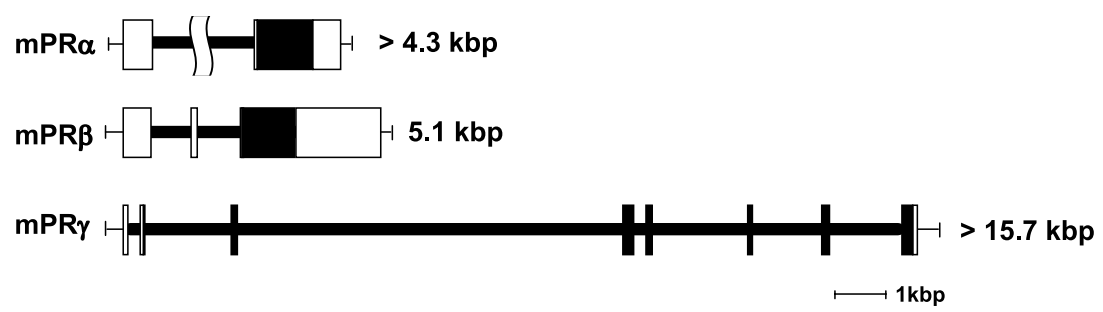

Figure 5 Schematic structures of the genes encoding IpmPR $\alpha, \beta$ and $\gamma$. Open boxes indicate untranslated regions of exons. Closed boxes indicate translated regions of exons. Bars represent introns.

assays. Expression of the IpmPR $\alpha$ gene was detected in all tissues analyzed. However relatively high expression was evident in all parts of the brain, pituitary, muscle and the testis. The IpmPR $\beta$ transcript was generally lower in abundance than IpmPR $\alpha$ and was observed primarily in parts of the brain (especially the hypothalamus and hindbrain), the pituitary, the ovary and the testis. Even though the IpmPR $\beta$ transcript was detected in other parts of the brain, gill, ventral aorta (including the surrounding thyroid follicles), and head kidney, abundance was very low. On the other hand, the IpmPR $\gamma$ transcript was mainly detected in the gill, ventral aorta, intestine, and trunk kidney with lower levels of expression in the cerebellum, hypothalamus and testis. The tissue expression of the $\mathrm{mPR} \alpha$ gene in zebrafish (DrmPR $\alpha$ ) and catfish differed. DrmPR $\alpha$ mRNA was detected primarily in zebrafish gonadal tissues and was less abundant than the transcript of DrmPR $\beta$. DrmPR $\beta$ was predominantly detected in the same tissues that the mPR $\beta$ transcript was found in catfish (i.e., brain, pituitary and gonadal tissues).

\section{Discussion}

In the present study, the cDNAs encoding three catfish putative mPRs, IpmPR $\alpha, \beta$ and $\gamma$, were isolated, and their secondary structures phylogenetic relationship to other mPR forms were determined. IpmPR $\alpha$ and $\beta$ showed relatively high identity at the amino acid sequence level, approximately $50 \%$, however, the $\gamma$ form shared less than 30\% identity with the other IpmPRs. Furthermore, phylogenetic analysis clearly placed each form of IpmPR together with their respective $\mathrm{mPR}$ subtype groups and showed greater divergence between the clusters of $\mathrm{mPR} \gamma \mathrm{s}$ compared with those of the $\alpha$ and $\beta$ forms. These findings strongly suggest that $\mathrm{mPR} \alpha$ and $\mathrm{mPR} \beta$ are closely related, whereas it is likely that

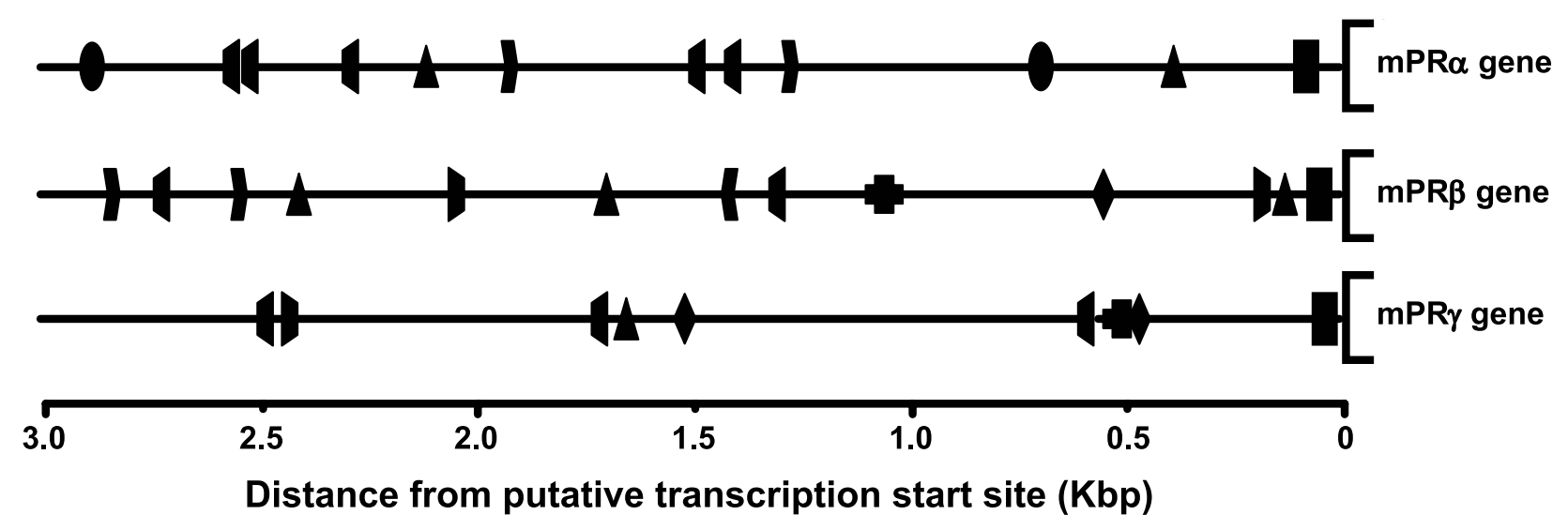

\section{$\checkmark$ AhR/Arnt OCRE Egr $\triangle$ SF-1 Dere Dgre/Preiare tata box}

Figure 6 Diagram of structures and potential transcription regulatory elements located in the $5^{\prime}$-flanking regions of the catfish mPR genes. AhR/Arnt, responsive element for aryl hydrocarbon receptor and its nuclear translocation factor; CRE, cAMP responsive element; Egr, binding site for early growth response genes; SF-1, steroidogenic factor-1 binding site; ERE, estrogen responsive element; GRE/PRE/ARE, glucocorticoid/progestin/androgen responsive element. 

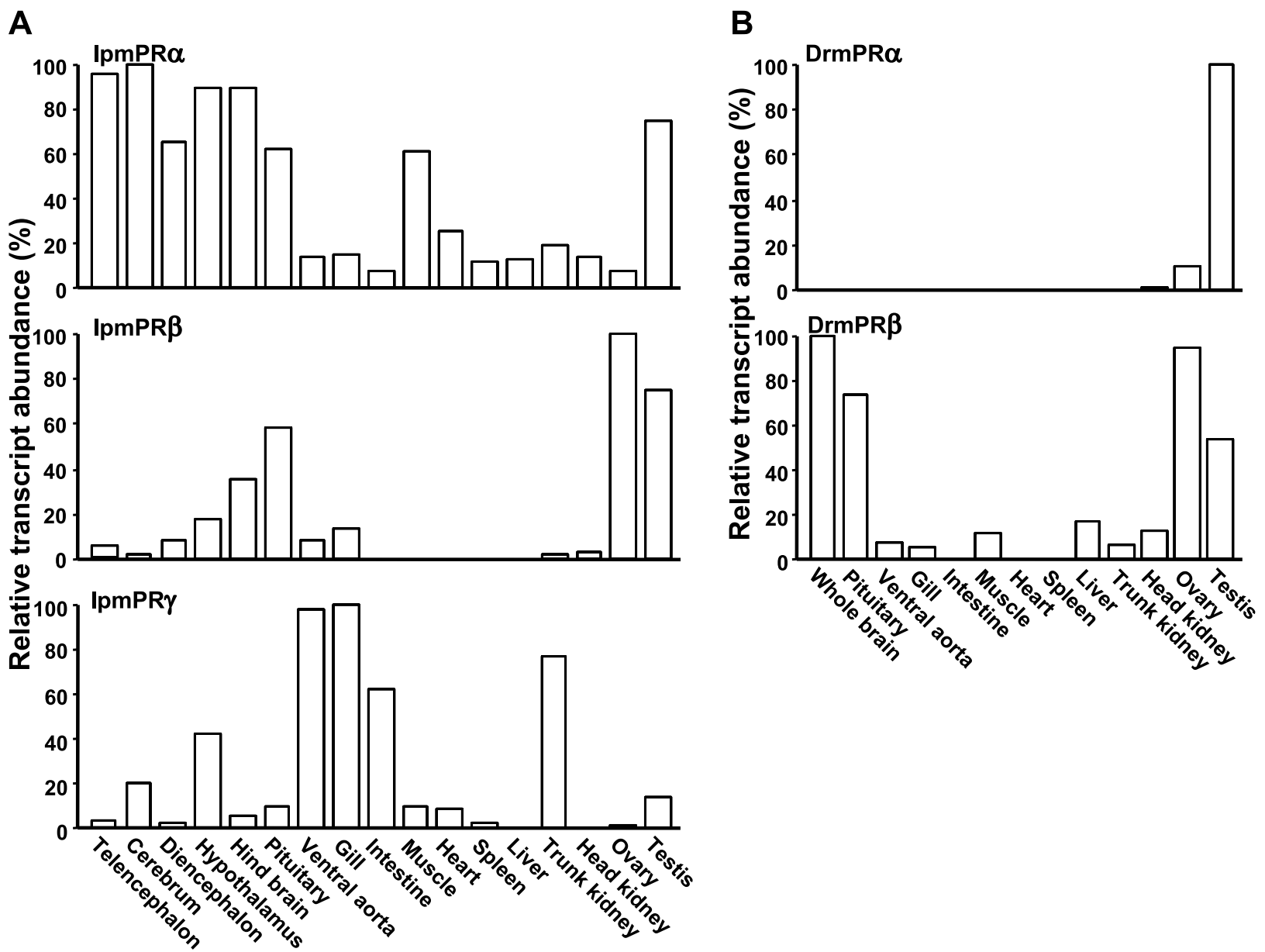

Figure 7 Tissue-specific expression of mPRs in (A) catfish and (B) zebrafish. The transcript abundance of each mPR was determined by real-time quantitative RT-PCR and normalized to the abundance of $\beta$-actin. Data are expressed as percentage of the transcript abundance of each mPR gene in the tissue representing the highest expression level.

$\mathrm{mPR} \gamma$ evolutionally diversified from other groups of mPRs.

Interestingly, six mPR-related sequences including $\operatorname{mPR} \alpha, \beta$ and $\gamma$, and three uncharacterized forms (FmPRLP 1-3) were retrieved from the Fugu genome database. Structure analysis of these novel forms demonstrated that two of them, FmPRLP 1 and 2, are closely related to $\mathrm{mPR} \alpha$, while the predicted amino acid residue of FmPRLP 3 shares high identity with the $\beta$ form. These conclusions are supported by the phylogenetic analysis showing their relationships to other mPRs. These findings suggest that FmPRLPs are potential members of $\mathrm{mPR}$ family, although further characterization of them is required, including the demonstration that their mRNAs are expressed in fish tissues and are functional genes. The mPRLPs are possibly species-specific or teleost-specific forms of mPR-related genes, since the human genome appears to contain only three forms of mPRs from this phylogenetic analysis. Further information on the number of mPR-related proteins in other animal species would provide valuable insight into the evolution of this new steroid receptor family.

The deduced amino acid sequences of each IpmPR showed high homology to other animal forms; in two regions in particular. The amino acid residues 69-84 and 273-287 in IpmPR $\alpha$ are quite highly conserved among all mPR forms, including FmPRLPs. The former conserved region contains a portion of the $\mathrm{N}$-terminal extra-cellular region and a potential N-linked glycosylation site identified in $\mathrm{mPR} \alpha$ and $\gamma$. These data may imply that these conserved regions are involved in fundamental functions of $\mathrm{mPR}$, for example, the ligand-binding, coupling with G-proteins.

Interestingly, IpmPR $\alpha$ and $\beta$ show comparable genomic organizations without any introns in their coding regions. In contrast, the structure of the $\mathrm{mPR} \gamma$ gene is quite different in that it includes six intron 
junctions within the coding region. This difference in the gene structure between IpmPR $\gamma$ and those of the $\alpha$ and $\beta$ forms appears to coincide with the secondary structural and phylogenetic divergence of the $\gamma$ from the $\alpha$ and $\beta$ forms. Furthermore, these features of genomic structure are completely conserved in the corresponding human and Fugu mPR genes. This finding strongly suggests that the mPR genes are phylogenetically conserved throughout the vertebrates based on a high degree of conservation of their genomic organization as well as their coding sequences.

The putative transcriptional regulatory elements located on the unique 5'-flanking regions of each IpmPR gene were also identified. 5'-flanking regions of each IpmPR show a TATA box within $90 \mathrm{bp}$ of the end of the $5^{\prime}$-UTR, which suggests that these 5 '-termini are close to the transcription start sites for each IpmPR gene. The 5'-flanking region of each IpmPR gene differentially exhibits the consensus regulatory elements for transcription factors related to reproductive physiology (CRE, SF-1 binding site; Morohashi et al. 1992), steroid hormone regulation, and cellular growth and differentiation (Egr; Tourtellotte et al. 1999 and AhR/Arnt; Poland \& Knutson 1982). Therefore, these transcription factors may be partly responsible for the direct transcriptional modulation of IpmPR genes. In fact, we recently have shown that the catfish $\mathrm{mPR} \alpha$ gene is down-regulated by a progestin, $17 \alpha, 20 \beta$-dihydroxy-4pregnene-3-one (the MIS in this species) while estradiol up-regulates its expression (Kazeto et al. 2005). Furthermore, it was reported that the protein abundance of mPR $\alpha$ was enhanced in seatrout ovary by hGG (Zhu et al. 2003a) whose effects are generally mediated by cAMP (Ascoli et al. 2002), although recent studies have shown that the catfish and zebrafish mPRs are not regulated by gonadotropins (Kazeto et al. 2005). The transcriptional regulatory elements identified in this study adds significantly to the paucity of information that is currently available concerning the gene regulation of mPRs and it provides valuable insights into the potential mechanisms of $\mathrm{mPR}$ gene regulation.

The different tissue distributions of the three IpmPR genes was expected based on the significant structural differences in their $5^{\prime}$-flanking regions. The transcript for IpmPR $\alpha$ was detected in all tissues examined whereas the $\beta$ form, although poorly expressed, was in greatest abundance in the brain, pituitary and gonads. The $\gamma$ form was primarily expressed in the posterior kidney, gill and intestine. The more sensitive RT-PCR procedure used in the present study demonstrates that the distribution of IpmPR $\alpha$ transcript is ubiquitous in catfish whereas Northern blot analysis only showed significant expression of the $\mathrm{mPR} \alpha$ gene in the brain, pituitary and gonads in seatrout (Zhu et al. 2003a), and in various reproductive tissues and the kidney in humans (Zhu et al. 2003b). Similarly, the RT-PCR procedure reveals that the $\mathrm{mPR} \beta$ gene is also expressed in the gonads in catfish, whereas only neural expression of the gene was detected in human tissues by Northern blot analysis (Zhu et al. 2003b). However, these two methods showed similar tissue expression of $\mathrm{mPR} \gamma$ genes in catfish and humans, with expression primarily in kidney and intestinal tissue (Zhu et al. 2003b). Interestingly, catfish gill also expresses $\operatorname{IpmPR} \gamma$, which suggests that IpmPR $\gamma$ may be involved in ion regulation.

In order to determine if the apparent differences seen in tissue-specific expression of $\mathrm{mPR} \alpha$ and $\beta$ in catfish are specific to this species, the tissue distribution of $\mathrm{mPR} \alpha$ and $\beta$ expression was examined in a second teleost species, the zebrafish. The tissue-specific gene expression of $\mathrm{mPR} \alpha$ differed in zebrafish, where the $\operatorname{mPR} \beta$ transcript was expressed almost exclusively in gonadal tissues. The apparent differences in tissue expression of the $\mathrm{mPR} \alpha$ gene among the different fish species investigated to date may indicate possible species-specific differences in the extra-gonadal functions of the $\mathrm{mPR} \alpha$ in fish. In contrast, the tissue-specific distribution of the $\mathrm{mPR} \beta$ transcript in zebrafish is essentially the same as in the catfish, that is in brain, pituitary and gonadal tissues, and potentially could reflect some conserved functions of mPR $\beta$ among these teleost species.

In conclusion, the cDNAs and the genes encoding three putative mPRs, the $\alpha, \beta$ and $\gamma$ forms, were isolated from catfish and structural analyses strongly suggest that the highly related $\alpha$ and $\beta$ forms of the catfish mPR are distantly related to the $\gamma$ form. Furthermore, structural analysis of the $5^{\prime}$-flanking regions of the $\mathrm{mPR}$ genes and the differences in the tissue-specific distributions of their transcripts suggest that expression of these genes is differentially modulated by multiple regulators in catfish. Currently, only a few studies have been published on this new class of steroid receptors. The molecular characteristics of the mPRs described in this study provide valuable insights into the possible functions and physiology of these novel steroid receptors.

\section{Acknowledgements}

This work was supported by a grant from the USDA (USDA: Enhancing Reproductive Efficiency; 00-352039105) and NSF (IBN-0214569) to JMT. YK was supported by a fellowship from the Japanese Society for the Promotion of Science. This is contribution number 05-104 from the Center of Marine Biotechnology.

\section{References}

Ascoli M, Fanelli F \& Segaloff DL 2002 The lutropin/ choriogonadotropin receptor, a 2002 perspective. Endocrine Reviews 23 141-174. 
Bagowski CP, Myers JW \& Ferrell JE Jr 2001 The classical progesterone receptor associates with $\mathrm{p} 42 \mathrm{MAPK}$ and is involved in phosphatidylinositol 3-kinase signaling in Xenopus oocytes. Fournal of Biological Chemistry 276 37708-37714.

Beato M 1989 Gene regulation by steroid hormones. Cell $\mathbf{5 6}$ 335-344.

Blom N, Gammeltoft S \& Brunak S 1999 Sequence- and structure-based prediction of eukaryotic protein phosphorylation sites. Fournal of Molecular Biology 294 1351-1362.

Breathnach R \& Chambon P 1981 Organization and expression of eukaryotic split genes coding for proteins. Annual Review of Biochemistry 50 349-383.

Falkenstein E, Tillmann HC, Christ M, Feuring M \& Wehling M 2000 Multiple actions of steroid hormones, a focus on rapid, nongenomic effects. Pharmacological Reviewes 52 513-556.

Gallo CJ, Hand AR, Jones TL \& Jaffe LA 1995 Stimulation of Xenopus oocyte maturation by inhibition of the G-protein alpha $\mathrm{S}$ subunit, a component of the plasma membrane and yolk platelet membranes. Fournal of Cellular Biology 130 275-284.

Hofmann K \& Stoffel W 1993 TMbase - a database of membrane spanning proteins segments. Biological Chemistry Hoppe-Seyler 374 166.

Kalinowski RR, Berlot CH, Jone TL, Ross LLF, Jaffe LA \& Mehlmann LM 2004 Maintenance of meiotic prophase in vertebrate oocytes by a $\mathrm{G}_{\mathrm{s}}$ protein mediated pathway. Developmental Biology 267 1-13.

Kazeto Y, Goto-Kazeto R, Place AR \& Trant JM 2004 Aromatase (CYP19A2) in zebrafish and channel catfish brains: Changes in expression associated with the reproductive cycle and endocrine disrupting chemicals. Fish Physiology and Biochemistry 28 29-32.

Kazeto Y, Goto-Kazeto R \& Trant JM 2005 Membrane-bound progestin receptors in channel catfish and zebrafish ovary: changes in expression associated with the reproductive cycles and hormonal reagents. General and Comparative Endocrinology (In press)

Losel R \& Wehling M 2003 Nongenomic actions of steroid hormones. Nature Reviewes Molecular Cell Biology 4 46-56.

Maller JL 1998 Recurring themes in oocyte maturation. Biologie Cellulaire 90 453-460.

Morohashi K, Honda S, Inomata Y, Handa H \& Omura T 1992 A common trans-acting factor, Ad4-binding protein, to the promoters of steroidogenic P-450s. Fournal of Biological Chemistry $26717913-17919$.

Poland A \& Knutson JC 1982 2,3,7,8-tetrachlorodibenzo-p-dioxin and related halogenated aromatic hydrocarbons: examination of the mechanism of toxicity. Annual Reviews of Pharmacology and Toxicology 22 517-554.
Saitou N \& Nei M 1987 The neighbor-joining method: a new method for reconstructing phylogenetic trees. Molecular Biology and Evolution 4 406-425.

Simoncini T \& Genazzani AR 2003 Non-genomic actions of sex steroid hormones. European fournal of Endocrinology 148 281-292.

Thomas P, Zhu Y \& Pace M 2002 Progestin membrane receptors involved in the meiotic maturation of teleost oocytes: a review with some new findings. Steroids $67511-517$.

Thomas P, Pang Y, Zhu, Y, Detweiler C \& Doughty K 2004 Multiple rapid progestin actions and progestin membrane receptor subtypes in fish. Steroids $69567-573$.

Tourtellotte WG, Nagarajan R, Auyeung A, Mueller C \& Milbrandt J 1999 Infertility associated with incomplete spermatogenic arrest and oligozoospermia in Egr4-deficient mice. Development 126 5061-5071.

Trant JM, Gavasso S, Ackers J, Chung BC \& Place AR 2001 Developmental expression of cytochrome P450 aromatase genes (CYP19a and CYP19b) in zebrafish fry (Danio rerio). Fournal of Experimental Zoology $290475-483$.

Tsai MJ \& O'Malley BW 1994 Molecular mechanisms of action of steroid/thyroid receptor superfamily members. Annual Reviews of Biochemistry 63 451-486.

Yamashita M, Mita K, Yoshida N \& Kondo T 2000 Molecular mechanisms of the initiation of oocyte maturation: general and species-specific aspects. Progress in Cell Cycle Research 4 115-129.

Yoshikuni M \& Nagahama Y 1994 Involvement of an inhibitory G-protein in the signal transduction pathway of maturation-inducing hormone $(17 \alpha$, $20 \beta$-dihydroxy-4-pregnen-3-one) action in rainbow trout (Oncorhynchus mykiss) oocytes. Developmental Biology 166 615-622.

Xu Y, Traystman RJ, Hurn PD \& Wang MM 2003 Neurite-localized estrogen receptor $\alpha$ mediates rapid signaling by estrogen. Fournal of Neuroscience Research 74 1-11.

Zhu Y, Rice CD, Pang Y, Pace M \& Thomas P 2003a Cloning, expression, and characterization of a membrane progestin receptor and evidence it is an intermediary in meiotic maturation of fish oocytes. PNAS $\mathbf{1 0 0} 2231-2236$.

Zhu Y, Bond J \& Thomas P 2003b Identification, classification, and partial characterization of genes in humans and other vertebrates homologous to a fish membrane progestin receptor. PNAS 100 2237-2242.

Received 9 December 2004

Accepted 10 February 2005

Made available online as an Accepted Preprint 11 February 2005 